\title{
State Estimation for Hybrid Systems: Applications to Aircraft Tracking *
}

\author{
Inseok Hwang*, Hamsa Balakrishnan ${ }^{\dagger}$, and Claire Tomlin ${ }^{\dagger}$ \\ *School of Aeronautics and Astronautics, Purdue University, IN 47907 \\ $\dagger$ Dept. of Aeronautics and Astronautics, Stanford University, CA 94305 \\ ihwang@purdue.edu, \{hamsa,tomlin\}@stanford.edu
}

\begin{abstract}
The problem of estimating the discrete and continuous state of a stochastic linear hybrid system, given only the continuous system output data, is studied. Well established techniques for hybrid estimation, known as the Multiple Model Adaptive Estimation algorithm, and the Interacting Multiple Model algorithm, are first reviewed. Conditions that must be satisfied in order to guarantee the convergence of these hybrid estimation algorithms are then presented. These conditions also provide a means to predict, as a function of the system parameters, which transitions in a hybrid system are relatively easy to detect. A new variant of hybrid estimation algorithms, called the Residual-Mean Interacting Multiple Model (RMIMM) algorithm, is then proposed and analyzed. The performance of RMIMM is demonstrated through multi-modal aircraft trajectory tracking examples.
\end{abstract}

\section{Introduction}

Hybrid systems are dynamical systems that combine continuous dynamics modeled by differential (or difference) equations and discrete dynamics modeled by finite automata. Since hybrid systems can suitably model the complex behavior of varied embedded control systems, such as robotic, transportation, and process control systems, there has been considerable interest in the estimation and control of hybrid systems among researchers in both academic and industrial communities $[1,2,3,4]$. The objective of hybrid estimation is to compute both the discrete and continuous state estimates of a hybrid system at any given time. Hybrid estimators usually consist of the combination of a bank of continuous state

${ }^{*}$ This research was supported by ONR under MURI contract N00014-02-1-0720, by NASA JUP under grant NAG2-1564, by NASA grant NCC2-5536, and by an NSF Career Award (ECS-9985072). H. Balakrishnan was supported by a Stanford Graduate Fellowship. 
estimators, each one designed for a different discrete state, or mode, and a mode selecting algorithm. How the correct mode is selected depends on the type of output data available. The hybrid estimators analyzed in this paper address a particularly challenging problem that of mode and continuous state estimation given only the observation of the continuous state. In this case, the hybrid estimators use the differences in statistical properties (such as mean and covariance) of the outputs of the different continuous state estimators to choose the correct mode.

In air traffic surveillance, the accurate tracking of aircraft is important because all traffic advisories are based on the current state estimates of the aircraft. In this domain, the observations correspond to radar measurements of the positions of the aircraft (observation of the continuous state). The flight mode of the aircraft, indicating, for example, constant velocity straight flight, or coordinate turn, is not observed. Yet knowledge of the flight mode would be valuable to a surveillance system, as it is one of the strongest indicators of the aircraft's future trajectory. For example, we consider an aircraft tracking problem, in which a possible flight trajectory of the aircraft is shown in Figure 1. The trajectory is composed of lines in which the aircraft flies straight and level at constant speed and circular arcs in which the aircraft maneuvers with different yet constant yaw rates. The more accurate and the faster the flight mode detection, the more accurate the aircraft trajectory prediction, leading to safe and efficient air traffic control.

A traditional continuous state estimator, such as a Kalman filter designed using a single linear aircraft model with constant system parameters, process and measurement noise, does not perform well when the aircraft changes its mode unexpectedly. This difficulty arises because the model on which the filter is based does not accurately represent the behavior of the aircraft over all of its flight regime. The flight mode changes in an aircraft depend on the pilot's input. In the aircraft tracking problems we consider in this paper, this input is usually unknown to the surveillance system. The lack of knowledge of the pilot's actions makes the flight mode changes of an aircraft nondeterministic because they cannot be determined $a$ priori. In this paper, we model this input as a random process. If a single linear continuous model is used for aircraft tracking, the process noise covariance in the model has to be large in order to account for model inaccuracy. This large process noise covariance leads to poor state estimates. A hybrid model with multiple modes that represent the flight regimes of an aircraft could represent the dynamics of the aircraft more accurately than one continuous model, and thus would give more accurate state estimates. In this paper, we model the dynamics of an aircraft as a discrete-time stochastic linear hybrid system whose modes correspond to the flight modes of the aircraft. The flight mode logic of the aircraft is represented by the discrete-state dynamics, and governed by a finite Markov chain. In this framework, aircraft tracking is a hybrid estimation problem that requires the computation 


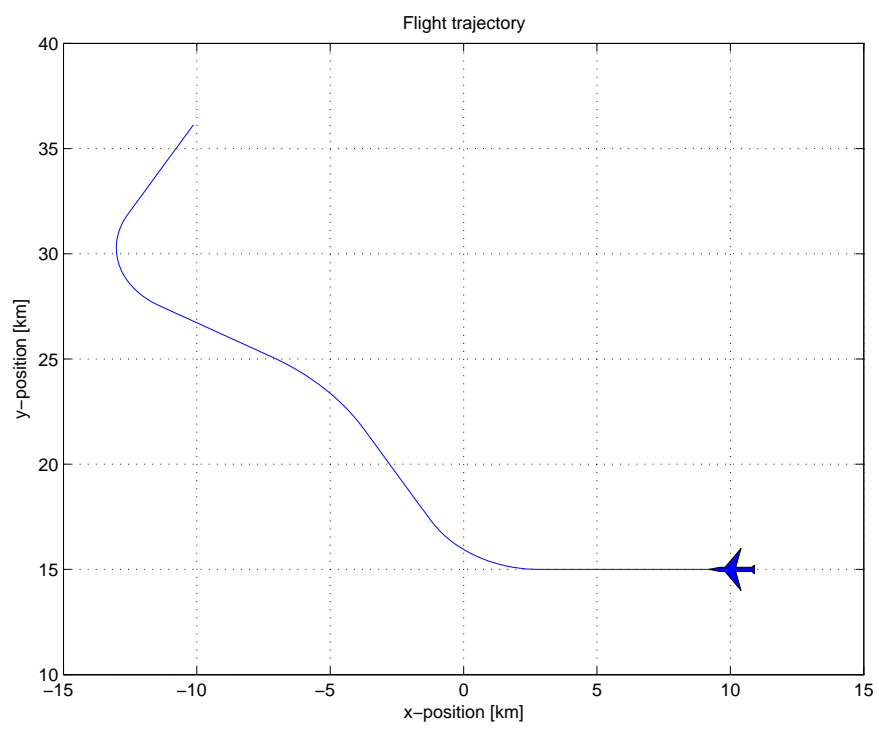

Figure 1: An aircraft trajectory.

of both the continuous state and discrete mode estimates. Therefore, in this paper, we consider a class of hybrid systems, called discrete-time stochastic linear hybrid systems, which have continuous dynamics modeled by linear difference equations and discrete-state dynamics modeled by a finite Markov chain.

\section{$1.1 \quad$ Related Work}

Hybrid estimation algorithms have been developed for discrete-time stochastic linear hybrid systems in which the mode transitions are governed by finite-state Markov chains. The Multiple Model Adaptive Estimation (MMAE) algorithm [5] is an algorithm in which, during hypothesis testing, the residuals of the different Kalman filters for each mode are used to form functions that reflect the likelihood that estimates of each of the different modes is the correct one. These functions, called likelihood functions, serve as adaptive weights, and the state estimate is the weighted sum of the state estimates computed by individual Kalman filters. In MMAE, the individual Kalman filters matched to the different modes run independently. In the Interacting Multiple Model (IMM) algorithm [6] which is a refinement of MMAE, a set of mode-matched Kalman filters interact with each other by using combinations of previous estimates computed by individual Kalman filters as initial conditions for each of the Kalman filters at every time step. The original rationale behind this refinement was to reduce the exponential complexity $\mathcal{O}\left(N^{T}\right)$ of the optimal hybrid estimator which minimizes the mean-square estimation error (where $N$ is the number of modes and $T$ is the total number of time samples) to $\mathcal{O}(N)$. Maybeck [5], Bar-Shalom et 
al. [6], Sworder and Boyd [7], and Hawkes and Moore [8] describe several similar hybrid estimation algorithms and their applications.

The performance of such hybrid estimators has been studied for several decades. Magill [9] provided sufficient conditions for the convergence of the adaptive weight for the correct mode to unity. These conditions are valid for the hybrid estimation of a specific class of systems, in which a constant parameter vector is unknown and the continuous dynamics in each mode has a single output. Lainiotis et al. $[10,11,12]$ extended these results to systems with multiple outputs, and derived the recursive form of the optimal adaptive estimator as well as its exact error covariance. Hawkes et al. [8] examined the asymptotic behavior of the adaptive weights which determine the performance of hybrid estimation algorithms. Many other approaches to the performance analysis of hybrid estimation, in which hypothesis testing is performed using adaptive weights, can be found in the work of Hawkes et al. [8], and the references therein. Baram et al. $[13,14]$ provided conditions under which, for a set of systems driven by stationary white Gaussian inputs and no discrete transitions, the mode probability of the true model converges to unity, i.e., the probability that the estimated model is the true model converges asymptotically to unity. However, the system studied by Baram et al. $[13,14]$ is a set of stochastic, stationary Gaussian models which run independently. Thus, the conditions derived in $[13,14]$ are more relevant to the observability of stochastic linear hybrid systems [15]. Caputi [16] derived a necessary condition for the convergence of hybrid estimation algorithms through the analysis of steady state residuals, and showed that the performance of the hybrid estimator depends on the DC gain of the continuous dynamics. This condition is only valid for a specific class of hybrid systems, in which the continuous dynamics for all the modes is the same, but the inputs are distinct and consist of a constant bias vector and zero-mean white Gaussian random noise.

The researchers cited above have analyzed hybrid estimation in several special classes of systems, yet general analysis techniques for evaluating the performance of hybrid estimation algorithms for stochastic linear hybrid systems have not been investigated in detail. In this paper, we analyze the properties of hybrid estimation algorithms for stochastic linear hybrid systems and derive conditions under which the computed hybrid estimates converge exponentially to the exact hybrid states. We say that a mode transition is more detectable than another if the time taken for the mode estimate to converge to the true mode is less for the former transition than for the latter. The results of this analysis give some insight into which mode transitions are more detectable than others and also into how to improve the performance of hybrid estimators. We then show analytically why the IMM algorithm, which has been widely and successfully used in the area of multiple target tracking, has better performance than the MMAE algorithm. Using the results of this performance analysis of hybrid estimation algorithms, we propose a modified IMM algorithm, called the 
Residual-Mean Interacting Multiple Model (RMIMM) algorithm, which uses the mean of the residual produced by each Kalman filter, producing better mode estimates as well as continuous state estimates than the standard IMM algorithm. We return to the aircraft tracking example to demonstrate the effectiveness of RMIMM.

This paper is organized as follows: Section 2 presents the structure of a stochastic linear hybrid system and problem statements. In Section 3, we describe a generic hybrid estimation algorithm, which could be MMAE, IMM, or any other refinement of the basic hybrid estimation algorithm. In Section 4, we analyze the performance of hybrid estimation algorithms and derive upper bounds for the mode estimation delay, as well as conditions that guarantee the exponential convergence of hybrid estimation algorithms. Section 4 also presents a comparison of the performance of different hybrid estimation algorithms. In Section 5, we derive the RMIMM algorithm and demonstrate its performance through a maneuvering aircraft tracking example.

\section{Discrete-time stochastic linear hybrid systems}

We consider a discrete-time stochastic linear hybrid system

$$
H:\left\{\begin{array}{cl}
x(k+1) & =A_{i} x(k)+B_{i} u(k)+w_{i}(k) \\
z(k) & =C_{i} x(k)+v_{i}(k)
\end{array}\right.
$$

where $k \in \mathbb{N}, x \in \mathbb{R}^{n}, u \in \mathbb{R}^{l}$ and $z \in \mathbb{R}^{p}$ are the continuous state, control input, and output variables respectively. The index $i \in\{1,2, \cdots, N\}$ represents the mode whose evolution is governed by the finite state Markov chain

$$
\mu(k+1)=\Pi \mu(k)
$$

where $\Pi=\left\{\pi_{i j}\right\} \in \mathbb{R}^{N \times N}$ is the mode transition matrix and $\mu(k) \in \mathbb{R}^{N}$ is the mode probability at time $k$. The system matrices $A_{i} \in \mathbb{R}^{n \times n}, B_{i} \in \mathbb{R}^{n \times l}$, and $C_{i} \in \mathbb{R}^{p \times n}$ for $i \in\{1,2, \cdots, N\}$ are assumed known. We denote the covariance of the initial state $x\left(k_{0}\right)$ as $\pi_{0} \in \mathbb{R}^{N}$, and assume that the process noise $w_{i}(k)$ and the measurement noise $v_{i}(k)$ are uncorrelated, zero-mean white Gaussian sequences with the covariance matrices $E\left[w_{i}(k) w_{i}(k)^{T}\right]=Q_{i} \in \mathbb{R}^{n \times n}$ and $E\left[v_{i}(k) v_{i}(k)^{T}\right]=R_{i} \in \mathbb{R}^{p \times p}$ respectively. $E[\cdot]$ and $(\cdot)^{T}$ denote expectation and matrix transpose respectively. It is assumed that $w_{i}(k)$ and $v_{i}(k)$ are both uncorrelated with the initial state, i.e., $E\left[x\left(k_{0}\right) w_{i}(k)^{T}\right]=E\left[x\left(k_{0}\right) v_{i}(k)^{T}\right]=0$. We define $Z(k)=\{z(0), \cdots, z(k)\}$ as the measurement sequence up to time $k$. Since the state evolution of a hybrid system has continuous trajectories as well as discrete jumps, we define a hybrid time trajectory: 
Definition 2.1 (Hybrid time trajectory) A hybrid time trajectory is a sequence of intervals $\left[k_{0}, k_{1}-1\right]\left[k_{1}, k_{2}-1\right] \cdots\left[k_{i}, k_{i+1}-1\right] \cdots$ where $k_{i}(i \geq 1)$ is the time at which the $i$-th mode transition occurs.

From the above definition, the sojourn time is defined as the time spent by the hybrid system between discrete state transitions. In this paper, by 'exponential convergence of a hybrid estimator' we mean:

Definition 2.2 (Exponential convergence of a hybrid estimator) Given a hybrid system $H$ with $N$ modes, we say that a hybrid estimator is exponentially convergent if its mode estimate exhibits correct identification of the mode transition sequence of the original system in a finite number of steps; the continuous state estimate at any instant has a unique mean and convergent covariance in the sense of the minimum mean square error; and the mean of the continuous state estimation error converges exponentially to the given steady-state error bound.

Definition 2.2 indicates the fact that a hybrid estimator converges exponentially only if the divergence of the mean of the continuous state estimation error when the mode estimate is incorrect does not destroy the exponential convergence of the mean of the estimation error when the mode estimate is correct.

In this paper, through the performance analysis of hybrid estimation algorithms, we address interesting problems: under what conditions the states estimates converge exponentially to the true states; how fast hybrid estimation algorithms can correctly detect mode transitions; and which mode transitions are easier to detect than others. Based on these analysis results, a new variant of hybrid estimation algorithms is proposed.

\section{$3 \quad$ Hybrid estimation algorithms}

In this section, we consider a generic hybrid estimation algorithm [5] for the discrete-time stochastic linear hybrid system (1)-(2). As seen in Section 1, such an algorithm typically contains a set of Kalman filters matched to the different modes of the hybrid system. Following the Bayesian estimation derivation in [5], the continuous state estimate is the conditional mean:

$$
\hat{x}(k+1)=E[x(k+1) \mid Z(k+1)]=\int_{-\infty}^{\infty} x(k+1) p(x(k+1) \mid Z(k+1)) d x(k+1)
$$

where $p(\cdot \mid \cdot)$ is the conditional probability density function, given by:

$$
p(x(k+1) \mid Z(k+1))=\sum_{i=1}^{N} \frac{p\left(x(k+1), Z(k+1), m_{i}(k+1)\right)}{p(Z(k+1))}
$$


and $m_{i}(k+1)$ denotes the event that the mode at time $k+1$ is $i$. Then, the state estimate (3) is

$$
\hat{x}(k+1)=\sum_{i=1}^{N} \hat{x}_{i}(k+1) p\left(m_{i}(k+1) \mid Z(k+1)\right)
$$

where $\hat{x}_{i}(k+1)=\int_{-\infty}^{\infty} x(k+1) p\left(x(k+1) \mid Z(k+1), m_{i}(k+1)\right) d x(k+1)$ is the modeconditioned state estimate of $x(k+1)$, which is the state estimate based on the assumption that the mode at time $k+1$ is $m_{i}(k+1)$ and is computed by the state estimator (in this paper, a Kalman filter) matched to mode $i$. Therefore, the state estimate (4) is a weighted sum of $N$ mode-conditioned state estimates produced by each Kalman filter with the weight $p\left(m_{i}(k+1) \mid Z(k+1)\right)$. The weight is given by

$$
p\left(m_{i}(k+1) \mid Z(k+1)\right)=\frac{\Lambda_{i}(k+1) p\left(m_{i}(k+1) \mid Z(k)\right)}{p(z(k+1) \mid Z(k))}
$$

where $\Lambda_{i}(k+1):=\mathcal{N}\left(r_{i}(k+1) ; 0, S_{i}(k+1)\right)$ is the likelihood function of mode $i$. $r_{i}(k+1)=z(k+1)-C_{i} \hat{x}_{i}(k+1 \mid k)$ is the residual produced by Kalman filter $i, S_{i}(k+1) \in \mathbb{R}^{p \times p}$ is the corresponding residual covariance, $\hat{x}_{i}(k+1 \mid k)$ is the state estimate by Kalman filter $i$ at time $k+1$ before the measurement update, and $\mathcal{N}(a ; b, c)$ is the probability at $a$ of a normal distribution with mean $b$ and covariance $c . p\left(m_{i}(k+1) \mid Z(k)\right)$ is the mode probability estimate at time $k+1$. If the mode transitions are governed by a finite Markov chain, the mode probability estimate can be expressed by

$$
p\left(m_{i}(k+1) \mid Z(k)\right)=\sum_{l=1}^{N} \pi_{i l} p\left(m_{l}(k) \mid Z(k)\right)
$$

Thus, the weight (5) is

$$
p\left(m_{i}(k+1) \mid Z(k+1)\right)=\frac{\Lambda_{i}(k+1)}{c(k+1)} \sum_{l=1}^{N} \pi_{i l} p\left(m_{l}(k) \mid Z(k)\right):=\mu_{i}(k+1)
$$

where $c(k+1)$ is a normalization constant. $\mu_{i}(k+1)$ in $(7)$ denotes the probability of mode $i$ at time $(k+1)$. The mode estimate at time $k$ is chosen to be the mode which has the maximum mode probability at that time. The mode probability depends not only on the finite Markov chain (discrete dynamics) but also on the likelihood produced by each Kalman filter (continuous dynamics). The state estimate (4) is

$$
\hat{x}(k+1)=\sum_{i=1}^{N} \hat{x}_{i}(k+1)\left[\frac{\Lambda_{i}(k+1)}{c(k+1)} \sum_{l=1}^{N} \pi_{i l} p\left(m_{l}(k) \mid Z(k)\right)\right]
$$


Equations (7)-(8) are the core of the Multiple Model Adaptive Estimation (MMAE) algorithm [5]. In MMAE, all individual Kalman filters run independently at every time step. Equations (7)-(8) show that the state estimate depends on the likelihood function; the performance of the hybrid estimator thus greatly depends on the behavior of the likelihood function. A variant of MMAE, the Interacting Multiple Model (IMM) algorithm [18]
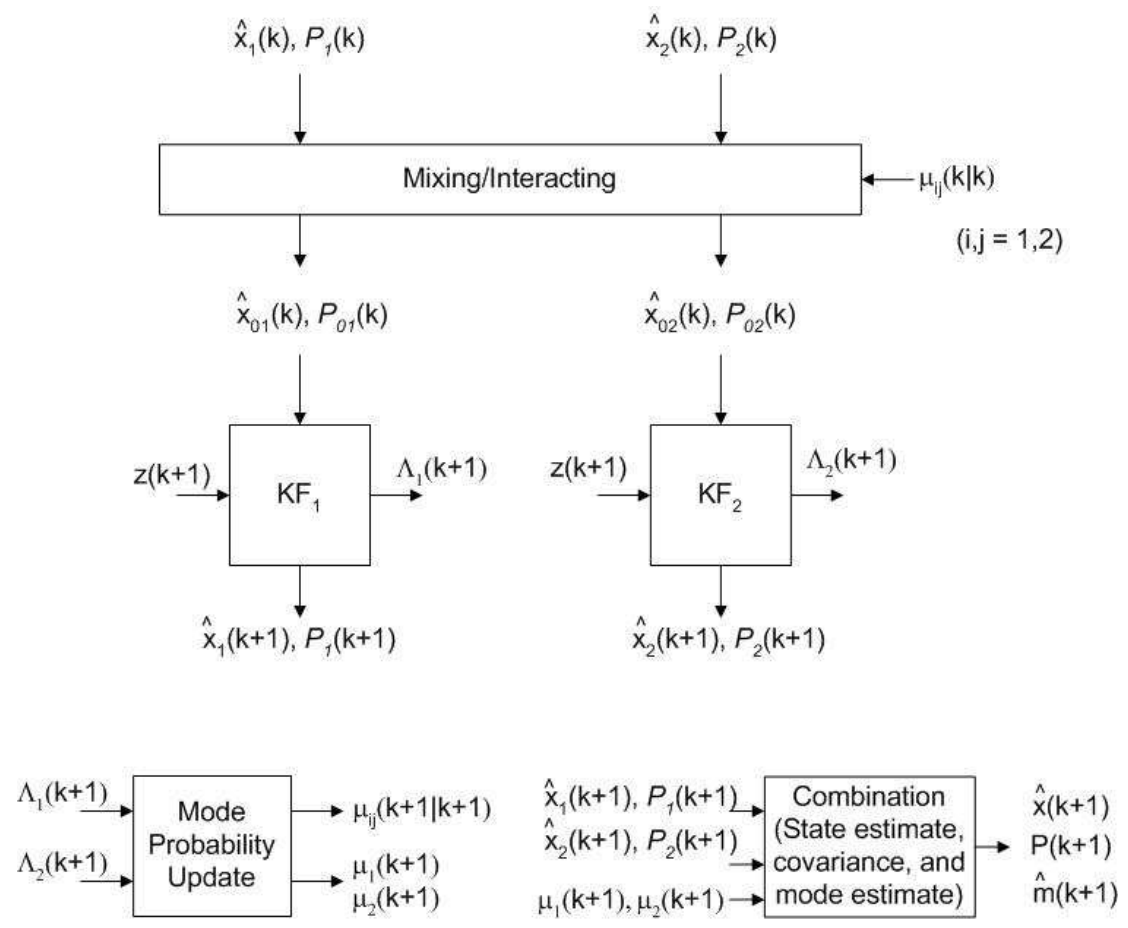

Figure 2: Structure of the IMM algorithm (two-mode system) from [17]

(on which RMIMM is based) has the same structure as MMAE except that it has a Mixing/Interacting step at the beginning of the estimation process, which computes new initial conditions for the Kalman filters matched to the individual modes at each time step. Figure 2 shows a schematic of the IMM algorithm for a system with two discrete modes. The IMM algorithm uses a bank of Kalman filters $\left(K F_{1}\right.$ and $\left.K F_{2}\right)$ and computes the mode probabilities $\mu_{i}(k+1)$ and the continuous state estimate $\hat{x}(k+1)$ in the same way as MMAE does in (7) and (8) respectively. However, individual Kalman filters share information about the other Kalman filters through new initial conditions $\left(\hat{x}_{0 i}(k), P_{0 i}(k)(\mathrm{i}=1,2)\right)$ at each time step. The components of IMM in Figure 2 are defined as follows:

1. Mixing probability: This is the probability that the system is in mode $i$ at time $k$, given that it is in mode $j$ at time $k+1(i, j \in\{1, \cdots, N\})$ :

$$
\mu_{i j}(k \mid k)=\frac{1}{c_{j}} \pi_{j i} \mu_{i}(k)
$$


where $c_{j}$ is a normalization constant, and where $\mu_{i}(k)$ is the mode probability of mode $i$ at time $k$, i.e., a measure of how probable it is that the system is in mode $i$ at time $k$. The initial condition $\mu_{i}(0)$ is assumed given, and is usually obtained from properties of the system.

2. New initial states and covariances: The input to each Kalman filter is adjusted by weighting the output of each Kalman filter with the mixing probability as the weight:

$$
\begin{aligned}
\hat{x}_{0 j}(k) & =\sum_{i=1}^{N} \hat{x}_{i}(k) \mu_{i j}(k \mid k) \\
P_{0 j}(k) & =\sum_{i=1}^{N}\left[P_{i}(k)+\left[\hat{x}_{i}(k)-\hat{x}_{0 j}(k)\right] \times\left[\hat{x}_{i}(k)-\hat{x}_{0 j}(k)\right]^{T}\right] \mu_{i j}(k \mid k)
\end{aligned}
$$

where $\hat{x}_{i}(k)$ and $P_{i}(k)$ are the state estimate and its covariance produced by Kalman filter $i$ after the measurement update at time $k$.

3. Kalman Filter: $N$ Kalman filters run in parallel (multiple-model-based (hybrid) estimation).

4. Mode likelihood functions: The likelihood function of mode $j$ is a measure of how likely it is that the model used in Kalman filter $j$ is the correct one; it is computed with the residual and its covariance produced by Kalman filter $j$ :

$$
\Lambda_{j}(k+1)=\mathcal{N}\left(r_{j}(k+1) ; 0, S_{j}(k+1)\right)
$$

where $r_{j}(k+1):=z(k+1)-C_{j} \hat{x}_{j}(k+1 \mid k)$ is the residual of Kalman filter $j$ and $S_{j}(k+1)$ is its covariance.

5. Mode probabilities: The probability of mode $j$ is a measure of how probable it is that the system is in mode $j$ :

$$
\mu_{j}(k+1)=\frac{1}{c(k+1)} \Lambda_{j}(k+1) \sum_{i=1}^{N} \pi_{j i} \mu_{i}(k)
$$

where $c(k+1)$ is a normalization constant. The probability of each mode is updated using the likelihood function.

6. Combination (output of the IMM algorithm): The state estimate is a weighted sum of the estimates from $N$ Kalman filters and the mode estimate is the mode which 
has the highest mode probability:

$$
\begin{aligned}
& \hat{x}(k+1)=\sum_{j=1}^{N} \hat{x}_{j}(k+1) \mu_{j}(k+1) \\
& P(k+1)=\sum_{j=1}^{N}\left\{P_{j}(k+1)+\left[\hat{x}_{j}(k+1)-\hat{x}(k+1)\right]\left[\hat{x}_{j}(k+1)-\hat{x}(k+1)\right]^{T}\right\} \mu_{j}(k+1) \\
& \hat{m}(k+1)=\arg \max _{j} \mu_{j}(k+1)
\end{aligned}
$$

where $\hat{m}(k+1)$ is the mode estimate at time $k+1$.

In the next section, we investigate the performance of hybrid estimation algorithms such as MMAE and IMM in detail.

\section{Performance analysis of the hybrid estimation algorithm}

In this section, we first analyze the performance of the hybrid estimation algorithm (either MMAE or IMM) by analyzing the steady-state mean residuals. Since steady-state analysis gives only necessary conditions on the performance of hybrid estimation, we then analyze the transient behavior of mode probabilities, which are functions of the likelihoods and therefore of the residuals.

\subsection{Performance derived from steady-state analysis}

Motivated by Caputi [16], we derive the steady-state mean residual for each mode $i$ for the hybrid system (1)-(2). We define:

$$
\begin{aligned}
\Delta A_{i} & :=A_{T}-A_{i}, \quad \Delta B_{i}:=B_{T}-B_{i} \\
\Delta C_{i} & :=C_{T}-C_{i}, \quad \hat{x}_{i_{s s}}:=\lim _{k \rightarrow \infty} \hat{x}_{i}(k) \\
\Delta u_{i_{s s}} & :=u_{T_{s s}}-u_{i_{s s}}:=\lim _{k \rightarrow \infty} u_{T}(k)-\lim _{k \rightarrow \infty} u_{i}(k) \\
\bar{e}_{i_{s s}} & :=\lim _{k \rightarrow \infty} E\left[e_{i}(k)\right]=\lim _{k \rightarrow \infty} E\left[\left(x(k)-\hat{x}_{i}(k)\right)\right]
\end{aligned}
$$

where the subscript $T \in\{1, \cdots, N\}$ represents the true mode. The mean residual and the mean estimation error of the filter $i(i \neq T)$ at time $k$ are

$$
\begin{aligned}
\bar{r}_{i}(k)= & C_{T} A_{T} \bar{e}_{i}(k-1)+\left(C_{T} \Delta A_{i}+\Delta C_{i} A_{T}-\Delta C_{i} \Delta A_{i}\right) \hat{x}_{i}(k-1) \\
& +\left(C_{T} \Delta B_{i}+\Delta C_{i} B_{T}-\Delta C_{i} \Delta B_{i}\right) u(k-1) \\
\bar{e}_{i}(k)= & \left(I-K_{i} C_{T}\right) A_{T} \bar{e}_{i}(k-1)+\left(\left(I-K_{i} C_{T}\right) \Delta A_{i}-K_{i} \Delta C_{i} A_{i}\right) \hat{x}_{i}(k-1) \\
& +\left(\left(I-K_{i} C_{T}\right) \Delta B_{i}-K_{i} \Delta C_{i} B_{i}\right) u(k-1)
\end{aligned}
$$


The steady-state mean residual for mode $i$ is

$$
\begin{aligned}
\bar{r}_{i_{s s}}:= & \lim _{k \rightarrow \infty} \bar{r}_{i}(k) \\
= & \left.\left(C_{T} A_{T} \Theta\left(\left(I-K_{i} C_{T}\right) \Delta A_{i}-K_{i} \Delta C_{i} A_{i}\right)\right)+\left(C_{T} \Delta A_{i}+\Delta C_{i} A_{T}-\Delta C_{i} \Delta A_{i}\right)\right) \hat{x}_{i_{s s}} \\
& +\left(C_{T} A_{T} \Theta\left(I-K_{i} C_{T}\right) B_{T}+C_{T} B_{T}\right) \Delta u_{i_{s s}}+\left(C_{T} A_{T} \Theta\left(\left(I-K_{i} C_{T}\right) \Delta B_{i}-K_{i} \Delta C_{i} B_{i}\right)\right. \\
& \left.+\left(C_{T} \Delta B_{i}+\Delta C_{i} B_{T}-\Delta C_{i} \Delta B_{i}\right)\right) u_{i_{s s}}
\end{aligned}
$$

where $\Theta:=\left(I-\left(I-K_{i} C_{T}\right) A_{T}\right)^{-1}$ and $K_{i}$ is the steady-state Kalman filter gain for mode $i$. We assume that $\Theta$ is invertible. If mode $i$ is the correct mode $(i=T)$, then $\bar{r}_{i_{s s}}=0$. If $\bar{r}_{j_{s s}} \neq 0(\forall j \neq i)$, then the correct mode can be detected because only the steady-state mean residual of the true mode is zero, and those of the other modes are not zero. However, even if mode $i$ is not the correct mode $(i \neq T)$, the steady-state mean residual for mode $i$ is zero if all of the following equalities are satisfied:

$$
\begin{array}{cc}
\left(I-K_{i} C_{T}\right) \Delta A_{i}-K_{i} \Delta C_{i} A_{i} & =0 \\
\left(C_{T} \Delta A_{i}+\Delta C_{i} A_{T}-\Delta C_{i} \Delta A_{i}\right) & =0 \\
\left(I-K_{i} C_{T}\right) \Delta B_{i}-K_{i} \Delta C_{i} B_{i} & =0 \\
\left(C_{T} \Delta B_{i}+\Delta C_{i} B_{T}-\Delta C_{i} \Delta B_{i}\right) & =0 \\
\Delta u_{i s s} & =0
\end{array}
$$

This means that if at least two models are identical and the corresponding control inputs are the same, then the steady-state residuals of both the corresponding modes are zero. In this case, the hybrid estimator cannot uniquely determine the correct mode. In other words, the performance of the hybrid estimation algorithm depends on the differences between the residuals which in turn arise from model differences and input differences. In the above condition, the first four equalities come from model differences and the last equality comes from input differences. This analysis result supports Maybeck's observation that the performance of MMAE depends on a significant difference between the residual characteristics $[5]$.

\subsection{Performance derived from transient analysis}

We now consider the transient mean behavior of a hybrid estimator, and analyze its performance in the sense of exponential convergence in Definition 2.2. A steady-state Kalman filter is assumed to be used as the state estimator for each mode. For the sake of notational simplicity, we define $\mu_{i}^{-}(k):=\sum_{l=1}^{N} \pi_{i l} \mu_{l}(k-1)$. The condition for correct mode detection at time $k$ is:

$$
\mu_{T}(k)>\mu_{i}(k), \quad \forall i \neq T
$$


Using $\mu_{i}(k):=p\left(m_{i}(k) \mid Z(k)\right)$ from $(7)$ and

$$
\Lambda_{i}(k)=\mathcal{N}\left(\bar{r}_{i}(k) ; 0, S_{i}\right):=(2 \pi)^{-n / 2}\left|S_{i}\right|^{-1} \exp \left[-\frac{1}{2} \bar{r}_{i}(k)^{T} S_{i}^{-1} \bar{r}_{i}(k)\right]
$$

in (5), where $S_{i}=S_{i}^{T}>0$ and $\bar{r}$ is the mean residual, (13) becomes

$$
0 \leq \bar{r}_{T}(k)^{T} S_{T}^{-1} \bar{r}_{T}(k)<\bar{r}_{i}(k)^{T} S_{i}^{-1} \bar{r}_{i}(k)+2 \ln \left(\frac{\left|S_{i}\right|}{\left|S_{T}\right|}\right)+2 \ln \left(\frac{\mu_{T}^{-}(k)}{\mu_{i}^{-}(k)}\right)
$$

To detect the correct mode exactly for any $k \in \mathbb{N}$, (14) must hold for all $k \in \mathbb{N}(\forall i \neq T)$. If there is a time delay $\left(\delta_{T}\right)$ for correct mode detection when a mode transition into mode $T$ occurs at time $k_{l}\left(l \in \mathbb{N}^{+}\right),(14)$ holds for $k \in\left[k_{l}+\delta_{T}, k_{l+1}\right)$. For the existence of an $\bar{r}_{T}(k)$ satisfying (14), using the properties of the eigenvalues of positive definite matrices [19], we derive the following:

Proposition 4.1 The correct mode can be detected in at most $\delta_{T}$ time steps after a mode transition at time $k_{l}$ if there exists $\delta_{T} \in \mathbb{N}^{+}$such that for $k \in\left[k_{l}+\delta_{T}, k_{l+1}\right)\left(l \in \mathbb{N}^{+}\right.$, $\forall i \neq T)$, Condition 1 holds, and either Condition 2 or Condition 3 is true.

$$
\begin{aligned}
& \text { 1. } \bar{r}_{i}(k)^{T} S_{i}^{-1} \bar{r}_{i}(k)+2\left[\ln \left(\frac{\left|S_{i}\right|}{\left|S_{T}\right|}\right)+\ln \left(\frac{\mu_{T}^{-}(k)}{\mu_{i}^{-}(k)}\right)\right]>0 \\
& \text { 2. } \bar{r}_{T}(k)^{T} S_{T}^{-1} \bar{r}_{T}(k)<\bar{r}_{i}(k)^{T} S_{i}^{-1} \bar{r}_{i}(k)+2\left[\ln \left(\frac{\left|S_{i}\right|}{\left|S_{T}\right|}\right)+\ln \left(\frac{\mu_{T}^{-}(k)}{\mu_{i}^{-}(k)}\right)\right] \\
& \text { 3. }\left\|\bar{r}_{T}(k)\right\|^{2}<\frac{\lambda_{\min }\left(S_{i}^{-1}\right)}{\lambda_{\max }\left(S_{T}^{-1}\right)}\left\|\bar{r}_{i}(k)\right\|^{2}+\frac{2}{\lambda_{\max }\left(S_{T}^{-1}\right)}\left[\ln \left(\frac{\left|S_{i}\right|}{\left|S_{T}\right|}\right)+\ln \left(\frac{\mu_{T}^{-}(k)}{\mu_{i}^{-}(k)}\right)\right]
\end{aligned}
$$

Conditions 1 and 2 indicate that the probability of the true mode should be greater than those of the other modes for correct mode detection. Since Condition 3 is obtained by considering the worst case, i.e., when the upper bound on the left-hand side of the inequality in Condition 2 is less than the lower bound on the right-hand side, Condition 3 is a sufficient condition for correct mode detection, and might be very conservative. However, it gives valuable insight into the performance of hybrid estimation. Fast mode detection is dependent not only on the magnitudes of the residuals produced by each Kalman filter but also on the residual covariances. If $\frac{\lambda_{\min }\left(S_{i}^{-1}\right)}{\lambda_{\max }\left(S_{T}^{-1}\right)}$ is small and/or $\frac{\left|S_{i}\right|}{\left|S_{T}\right|}$ is small, it is difficult for Condition 3 to hold and thus to detect the correct mode. Therefore, by checking the eigenvalues and determinant of $S_{i}^{-1}$, we can tell which mode transitions are more detectable than the others. This is similar to the idea of the observability grammian as a measure of which states are more observable than others [20]. If we consider the steady-state residual mean, Condition 3 becomes, $\forall i \neq T$,

$$
\left\|\bar{r}_{T_{s s}}\right\|^{2}<\frac{\lambda_{\min }\left(S_{i}^{-1}\right)}{\lambda_{\max }\left(S_{T}^{-1}\right)}\left\|\bar{r}_{i_{s s}}\right\|^{2}+\frac{2}{\lambda_{\max }\left(S_{T}^{-1}\right)}\left[\ln \left(\frac{\left|S_{i}\right|}{\left|S_{T}\right|}\right)+\ln \left(\frac{\mu_{T_{s s}}^{-}}{\mu_{i_{s s}}^{-}}\right)\right]
$$


Therefore, if the asymptotic behavior of the residuals satisfies (15) and the sojourn times are long enough for the residuals to converge to their steady-state values, then MMAE is guaranteed to estimate the hybrid states correctly.

We now derive the mode estimation delay $\delta_{i}$ using Condition 3 in Proposition 4.1. The mean residual of the correct filter at time $k_{l}+\delta_{i}\left(l \in \mathbb{N}^{+}\right)$, when $i=T$, is $\bar{r}_{T}\left(k_{l}+\delta_{T}\right)=$ $C_{T} A_{T}\left[\left(I-K_{T} C_{T}\right) A_{T}\right]^{\delta_{T}-1} \bar{e}_{T}\left(k_{l}\right)$, where $\bar{e}_{T}\left(k_{l}\right)$ is the mean of the estimation error of the correct filter at time $k_{l}$. For the sake of notational simplicity, we define

$$
\begin{aligned}
& F_{T}:=\left(I-K_{T} C_{T}\right) A_{T}, F_{i}:=\left(I-K_{i} C_{T}\right) A_{T} \\
& H_{i}^{x}:=C_{T} \Delta A_{i}+\Delta C_{i} A_{T}-\Delta C_{i} \Delta A_{i} \\
& H_{i}^{u}:=C_{T} \Delta B_{i}+\Delta C_{i} B_{T}-\Delta C_{i} \Delta B_{i} \\
& G_{i}^{x}:=\left(I-K_{i} C_{T}\right) \Delta A_{i}-K_{i} \Delta C_{i} A_{i} \\
& G_{i}^{u}:=\left(I-K_{i} C_{T}\right) \Delta B_{i}-K_{i} \Delta C_{i} B_{i} \\
& L_{i}:=\left[\begin{array}{lll}
C_{T} A_{T} & H_{i}^{x} & H_{i}^{u}
\end{array}\right]
\end{aligned}
$$

The norm of the mean residual of the correct filter at time $k_{l}+\delta_{T}$ is

$$
\left\|\bar{r}_{T}\left(k_{l}+\delta_{T}\right)\right\| \leq \bar{\sigma}\left(C_{T} A_{T}\right) \bar{\sigma}\left(F_{T}\right)^{\delta_{T}-1}\left\|\bar{e}_{T}\left(k_{l}\right)\right\|
$$

where $\bar{\sigma}(\cdot)$ denotes the maximum singular value. Similarly, we can show that the norm of the mean residual of the incorrect filter at time $k_{l}+\delta_{T}$ is

$$
\left\|\bar{r}_{i}\left(k_{l}+\delta_{T}\right)\right\| \geq \underline{\sigma}\left(L_{i}\right) \underline{\sigma}\left(\left[\begin{array}{lll}
F_{i} & G_{i}^{x} & G_{i}^{u}
\end{array}\right]\right)^{\delta_{T}-1}\left\|\bar{e}_{i}\left(k_{l}\right)\right\|
$$

where $\underline{\sigma}(\cdot)$ denotes the minimum singular value, and $\bar{e}_{i}\left(k_{l}\right)$ is the mean estimation error of the incorrect filter at time $k_{l}$. We define

$$
\begin{aligned}
\alpha & :=\frac{\lambda_{\min }\left(S_{i}^{-1}\right)}{\lambda_{\max }\left(S_{T}^{-1}\right)} \\
\beta(k) & :=\frac{2}{\lambda_{\max }\left(S_{T}^{-1}\right)}\left[\ln \left(\frac{\left|S_{i}\right|}{\left|S_{T}\right|}\right)+\ln \left(\frac{\mu_{T}^{-}(k)}{\mu_{i}^{-}(k)}\right)\right]
\end{aligned}
$$

Substituting this along with (17) and (18) in Condition 3 of Proposition 4.1, we obtain the following condition:

$\bar{\sigma}\left(C_{T} A_{T}\right)^{2} \bar{\sigma}\left(F_{T}\right)^{2\left(\delta_{T}-1\right)}\left\|\bar{e}_{T}\left(k_{l}\right)\right\|^{2}<\beta\left(k+\delta_{T}\right)+\alpha \underline{\sigma}\left(L_{i}\right)^{2} \underline{\sigma}\left(\left[\begin{array}{ccc}F_{i} & G_{i}^{x} & G_{i}^{u}\end{array}\right]\right)^{2\left(\delta_{T}-1\right)}\left\|\bar{e}_{i}\left(k_{l}\right)\right\|^{2}$

To find $\delta_{T}$ explicitly, we alternatively write the mean residual of the incorrect filter at time $k_{l}+\delta_{T}, \bar{r}_{i}\left(k_{l}+\delta_{T}\right)$ as: 


$$
\begin{aligned}
\bar{r}_{i}\left(k_{l}+\delta_{T}\right)= & C_{T} A_{T} F_{i}^{\delta_{T}-1} \bar{e}_{i}\left(k_{l}\right)+H_{i}^{x} \hat{x}_{i}\left(k_{l}+\delta_{T}-1\right) \\
& +C_{T} A_{T}\left[F_{i}^{\delta_{T}-2} G_{i}^{x} \hat{x}_{i}\left(k_{l}\right)+\cdots+G_{i}^{x} \hat{x}_{i}\left(k_{l}+\delta_{T}-2\right)\right] \\
& +C_{T} A_{T}\left[F_{i}^{\delta_{T}-2} G_{i}^{u} u\left(k_{l}\right)+\cdots+G_{i}^{u} u\left(k_{l}+\delta_{T}-2\right)\right]+H_{i}^{u} u\left(k_{l}+\delta_{T}-1\right)
\end{aligned}
$$

We denote the last four terms of (20) by $b_{i}\left(k_{l}+\delta_{T}-1\right)$ and $J_{i}\left(k_{l}+\delta_{T}\right):=\alpha \| b_{i}\left(k_{l}+\delta_{T}-\right.$ $2) \|^{2}+\beta\left(k_{l}+\delta_{T}\right)$. Using this in Condition 3 of Proposition 4.1 and combining it with (19), we get:

Proposition 4.2 The correct mode can be detected $\delta_{T}$ time steps after a mode transition if Condition 1 of Proposition 4.1 holds and there exists $\delta_{T} \in \mathbb{N}^{+}, \delta_{T}<k_{l+1}-k_{l}, l \in \mathbb{N}^{+}$, $\forall i \neq T$, such that either of the following conditions is true.

1. $\bar{\sigma}\left(C_{T} A_{T}\right)^{2} \bar{\sigma}\left(F_{T}\right)^{2\left(\delta_{T}-1\right)}\left\|\bar{e}_{T}\left(k_{l}\right)\right\|^{2}<\beta\left(k+\delta_{T}\right)+\alpha \underline{\sigma}\left(L_{i}\right)^{2} \underline{\sigma}\left(\left[\begin{array}{ccc}F_{i} & G_{i}^{x} & G_{i}^{u}\end{array}\right]\right)^{2\left(\delta_{T}-1\right)}\left\|\bar{e}_{i}\left(k_{l}\right)\right\|^{2}$

2. $\delta_{T}>1+\left\{2 \ln \left[\frac{\underline{\sigma}\left(F_{i}\right)}{\overline{\bar{\sigma}}\left(F_{T}\right)}\right]\right\}^{-1}\left\{-\ln \alpha+2 \ln \left[\frac{\bar{\sigma}\left(C_{T} A_{T}\right)}{\underline{\underline{\sigma}\left(C_{T} A_{T}\right)}}\right]+2 \ln \left[\frac{\left\|\bar{e}_{T}\left(k_{l}\right)\right\|}{\left\|\bar{e}_{i}\left(k_{l}\right)\right\|}\right]\right\}$, when $J_{i}\left(k_{l}+\delta_{T}\right) \geq 0$.

Proposition 4.2 indicates that for correct mode detection in at most $\delta_{T}$ time steps after a mode transition, the mean residual of the correct filter should be less than that of the incorrect filters. Although the actual value of $\beta\left(k_{l}+\delta_{T}\right)$, given by

$$
\beta\left(k_{l}+\delta_{T}\right)=\frac{2}{\lambda_{\max }\left(S_{T}^{-1}\right)}\left[\ln \left(\frac{\left|S_{i}\right|}{\left|S_{T}\right|}\right)+\ln \left(\frac{\mu_{T}^{-}\left(k_{l}+\delta_{T}\right)}{\mu_{i}^{-}\left(k_{l}+\delta_{T}\right)}\right)\right]
$$

might be negative, its magnitude is given in a logarithmic scale, and thus, $J_{i}\left(k_{l}+\delta_{T}\right) \geq 0$ is easily satisfied. Condition 2 in Proposition 4.2 provides a source of intuition on the performance of hybrid estimation algorithms. For a small mode estimation delay, the following must be small if $J_{i}\left(k_{l}+\delta_{T}\right) \geq 0:(\forall T \in\{1, \cdots, N\}, \forall i \neq T)$,

$$
\frac{1}{2} \log \left(\frac{\lambda_{\max }\left(S_{T}^{-1}\right)}{\lambda_{\min }\left(S_{i}^{-1}\right)}\right)+\log \left[\frac{\bar{\sigma}\left(C_{T} A_{T}\right)}{\underline{\sigma}\left(C_{T} A_{T}\right)}\right]+\log \left[\frac{\left\|\bar{e}_{T}\left(k_{l}\right)\right\|}{\left\|\bar{e}_{i}\left(k_{l}\right)\right\|}\right]
$$

where mode $T$ is the correct mode after the mode transition at time $k_{l}\left(l \in \mathbb{N}^{+}\right)$. Firstly, $\frac{\lambda_{\max }\left(S_{T}^{-1}\right)}{\lambda_{\min }\left(S_{i}^{-1}\right)}$ must be small. Here, the residual covariance $S_{i}$ computed by Kalman filter $i$ satisfies the algebraic Riccati equation. Therefore, $\frac{\lambda_{\max }\left(S_{T}^{-1}\right)}{\lambda_{\min }\left(S_{i}^{-1}\right)}$ depends only on the system parameters $A_{i}, C_{i}, Q_{i}, R_{i}$ and $A_{T}, C_{T}, Q_{T}, R_{T}$. Thus, by checking the residual covariance matrices for each Kalman filter which can be done without any measurements, we can tell which mode transition is more detectable than the others. Secondly, if the condition number of $C_{T} A_{T}$ is close to 1 , the second term in (21) becomes small. Thus, we also say which mode is more easily estimated than the others by checking the condition number of $C_{T} A_{T}$ 
for all $T$. Thirdly, $\frac{\left\|\bar{e}_{T}\left(k_{l}\right)\right\|}{\left\|\bar{e}_{i}\left(k_{l}\right)\right\|}$ must be small, i.e., the mean state estimation errors produced by mode-mismatched Kalman filters should be small and close to the error produced by the correct Kalman filter.

For a system with two modes, if we assume that the estimator converges between transitions and that the mode transition matrix $\Pi$ is diagonally dominant, we obtain the following condition:

Proposition 4.3 For a hybrid system with two modes, the correct mode can be detected $\delta_{T}$ time steps after a mode transition if there exists $\delta_{T} \in \mathbb{N}^{+}, \delta_{T}<k_{l+1}-k_{l}, l \in \mathbb{N}^{+}, i \neq T$, such that

$$
\begin{aligned}
& \alpha \underline{\sigma}\left(L_{i}\right)^{2} \underline{\sigma}\left(\left[\begin{array}{ccc}
F_{i} & G_{i}^{x} & G_{i}^{u}
\end{array}\right]\right)^{2\left(\delta_{T}-1\right)}\left\|\bar{e}_{i}\left(k_{l}\right)\right\|^{2}+\frac{2}{\lambda_{\max }\left(S_{T}^{-1}\right)}\left[\ln \left(\frac{\left|S_{i}\right|}{\left|S_{T}\right|}\right)-\ln \left(\frac{\pi_{i i}}{1-\pi_{i i}}\right)\right] \\
> & \bar{\sigma}\left(C_{T} A_{T}\right)^{2} \bar{\sigma}\left(F_{T}\right)^{2\left(\delta_{T}-1\right)}\left\|\bar{e}_{T}\left(k_{l}\right)\right\|^{2}
\end{aligned}
$$

Proposition 4.3 implies that if (22) is satisfied, i.e, the mean residual of the correct mode is less than that of the other mode, then the mode probability of the correct mode is greater than that of the other mode $\delta_{T}$ time steps after a mode transition at time $k_{l}$. Thus, the correct mode is detected $\delta_{T}$ time steps after a mode transition.

Now, we consider the conditions under which the mode change detection is instantaneous. Assuming that the time between mode transitions is sufficient to allow the Kalman filters and mode probabilities to converge, we obtain, for a system with $N$ modes:

Proposition 4.4 The correct mode is detected instantaneously if the following condition holds:

$$
\begin{aligned}
& \alpha \underline{\sigma}\left(L_{i}\right)^{2} \underline{\sigma}\left(\left[\begin{array}{ccc}
F_{i} & G_{i}^{x} & G_{i}^{u}
\end{array}\right]\right)^{2}\left\|\bar{e}_{i}\left(k_{l}-1\right)\right\|^{2}+\frac{2}{\lambda_{\max }\left(S_{T}^{-1}\right)}\left[\ln \left(\frac{\left|S_{i}\right|}{\left|S_{T}\right|}\right)+\ln \left(\frac{\pi_{j T}}{\pi_{j j}}\right)_{\min }\right] \\
> & \bar{\sigma}\left(C_{T} A_{T}\right)^{2}\left\|\bar{e}_{T}\left(k_{l}-1\right)\right\|^{2}
\end{aligned}
$$

where $\left(\frac{\pi_{j T}}{\pi_{j j}}\right)_{\text {min }}$ is the smallest ratio of off-diagonal to diagonal elements in any row of the $N \times N$ transition matrix.

Proposition 4.4 provides a means to test if the correct mode can be detected without delay after a mode transition.

Finally, we present the conditions to guarantee exponential convergence of the hybrid estimator once the correct mode sequence has been detected [15].

Theorem 4.1 (Theorem 3 from [15]): Consider a given stochastic linear hybrid system, an error convergence set $M_{0}$ and rate of convergence $\zeta,|\zeta|<1,\left|\alpha\left(A_{i}-K_{i} C_{i}\right)\right| \leq|\zeta|$ for all $i=1 \ldots N$, where $\alpha(A)$ is the maximal absolute value of the eigenvalues of $A$. Let $\kappa(A)=\|\Gamma\|\left\|\mid \Gamma^{-1}\right\|$, the condition number of $A$ under the inverse, where $\Gamma^{-1} A \Gamma=\mathcal{J}$, the Jordan canonical form. Then, if the following seven conditions are satisfied: 
1. The system is observable in the sense of a hybrid system [15].

2. $\left\{A_{i}, C_{i}\right\}$ couples are observable for all $i=1 \ldots N$.

3. $\left\{A_{i}, \Gamma_{i}^{1 / 2}\right\}$ couples are controllable for all $i=1 \ldots N$.

4. $\left(A_{i}-K_{i} C_{i}\right)$ is stable for all $i=1 \ldots N$ with all distinct eigenvalues.

5. There exists $X>0$ such that $\|x(k)\|_{\infty} \leq X, i, j=1 \ldots N, k=1,2, \ldots$ such that

$$
\begin{aligned}
& \left\|\left[\left(A_{i}-A_{j}\right)-K_{i}\left(C_{i}-C_{j}\right)\right] \bar{x}(k)\right\|_{\infty} \\
\leq & U=\max \left\|\left(A_{i}-A_{j}\right)-K_{i}\left(C_{i}-C_{j}\right)\right\|_{1} X
\end{aligned}
$$

6. The maximum mode estimation delay, $\delta$ satisfies the relation

$$
\delta \leq \frac{M_{0}}{\sqrt{n} U \max \left[\kappa\left(A_{i}-K_{i} C_{i}\right)\right]}
$$

7. The minimum time between mode transitions, $\tilde{\Delta}$, known as the minimum sojourn time, satisfies the conditions

$$
\begin{aligned}
& \tilde{\Delta}>\beta_{\min }+\delta \text {, where } \\
& \begin{aligned}
\beta_{\text {min }}> & \max \left[\frac{1}{|\log \zeta|} \log \left|\left(1-\frac{\sqrt{n} U \delta \kappa\left(A_{i}-K_{i} C_{i}\right)}{M_{0}}\right)\right|,\right. \\
& \left.\max \frac{\log \left(\kappa\left(A_{i}-K_{i} C_{i}\right)\right)}{\left|\log \left(\alpha\left(A_{i}-K_{i} C_{i}\right)\right)\right|}\right]
\end{aligned}
\end{aligned}
$$

Then, a hybrid estimator can be designed that converges to the set $M_{0}$ with a rate of convergence greater than or equal to $\zeta$.

Proof See [15].

Along with Theorem 4.1, Propositions 5.1-5.4 present the conditions under which, in the event of a mode detection delay $\delta$, the sojourn time is long enough for the error convergence during the period of correct detection $(\tilde{\Delta}-\delta)$ to balance the divergence of the error during the mode mismatch. From the present results, we can evaluate the performance of a given hybrid estimator and also find the minimum sojourn time required in each mode to guarantee exponential convergence of the mean-square estimation error.

\subsection{Performance comparisons}

We use the mode estimation delay as a performance metric for comparison of the MMAE and IMM algorithms since a small mode estimation delay usually corresponds to a small estimation error. Analyzing Condition 2 of Proposition 4.2, (or (21)), we can explain the 
performance of hybrid estimation algorithms qualitatively. Since Condition 2 of Proposition 4.2 shows that small $\frac{\lambda_{\max }\left(S_{T}^{-1}\right)}{\lambda_{\min }\left(S_{i}^{-1}\right)}$ leads to a small mode estimation delay, $\frac{\lambda_{\max }\left(S_{T}^{-1}\right)}{\lambda_{\min }\left(S_{i}^{-1}\right)}$ is indicative of which mode transition is easier to detect than the others. In addition, $\frac{\lambda_{\max }\left(S_{T}^{-1}\right)}{\lambda_{\min }\left(S_{i}^{-1}\right)}$ is a function of system parameters such as $Q_{i}, R_{i}, Q_{T}$ and $R_{T}$ which are design parameters for the Kalman filters $i$ and $T$. Thus, we can make $\frac{\lambda_{\max }\left(S_{T}^{-1}\right)}{\lambda_{\min }\left(S_{i}^{-1}\right)}$ small by adjusting these parameters (Kalman filter tuning) and thus reduce the mode estimation delay. For a small estimation delay, $\frac{\left\|\bar{e}_{T}\left(k_{l}\right)\right\|}{\left\|\bar{e}_{i}\left(k_{l}\right)\right\|}$ in $(21)$ should be small and this holds when the mean state estimation errors computed by incorrect Kalman filters are close to that of the correct Kalman filter. The mixing step in IMM was originally devised to reduce the complexity of

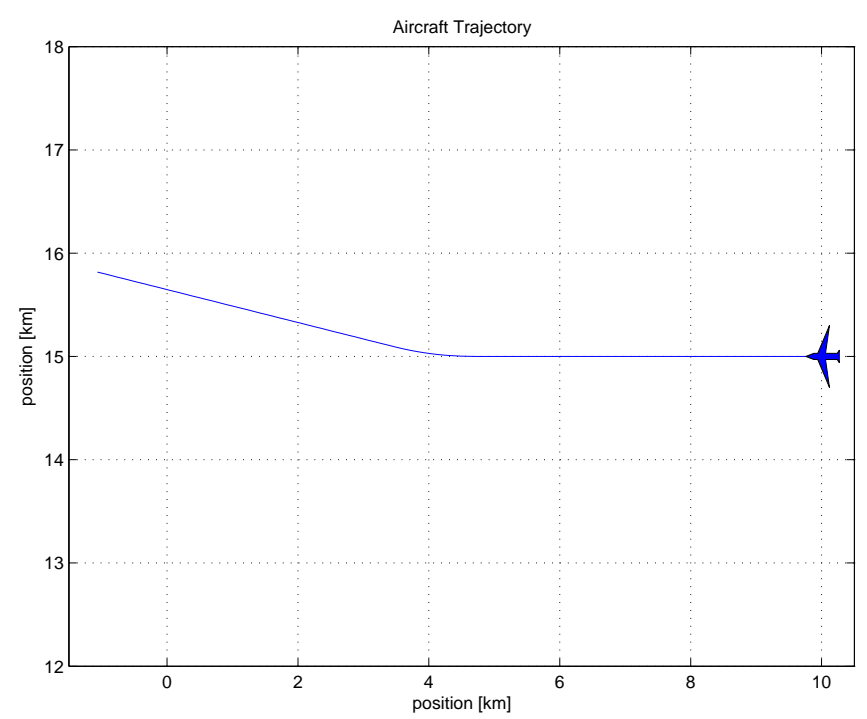

Figure 3: An aircraft trajectory.

the algorithm, yet it also keeps the estimation errors due to filter mismatch small. At the mixing step at each time instant, IMM shifts the initial conditions for each Kalman filter closer to the (correct) estimate computed by IMM at the previous time step. Therefore, the means of the state estimation errors produced by the incorrect Kalman filters are close to that of the correct Kalman filter. The mode estimation delay of IMM is therefore smaller than that of MMAE which does not have this mixing mechanism, and translates to better performance. Maybeck [5] proposes two ad hoc methods to improve adaptability of MMAE: enforcing a lower bound on the mode probabilities and adding extra noise to the the Kalman filter models. IMM does both inherently.

We now illustrate this through an aircraft tracking example, with two discrete modes, the constant velocity (CV) mode and the coordinated turn (CT) mode. In this example, we use a discrete-time stochastic linear hybrid system with two modes as the aircraft model. 
This hybrid model is different from the typical linear aircraft model (e.g., $(4.9,19)$ in [21]) used for the analysis and synthesis of aircraft control systems. The hybrid model does not need information on system parameters while the accuracy of the linear model is strongly dependent on these parameters whose accurate values are not usually available in the aircraft tracking problems. The dynamics of the hybrid model are given by

$$
\begin{aligned}
x(k+1) & =\left[\begin{array}{llll}
1 & T & 0 & 0 \\
0 & 1 & 0 & 0 \\
0 & 0 & 1 & T \\
0 & 0 & 0 & 1
\end{array}\right] x(k)+\left[\begin{array}{cc}
\frac{T^{2}}{2} & 0 \\
T & 0 \\
0 & \frac{T^{2}}{2} \\
0 & T
\end{array}\right] u_{i}(k)+w_{i}(k) \\
y(k) & =\left[\begin{array}{llll}
1 & 0 & 0 & 0 \\
0 & 0 & 1 & 0
\end{array}\right] x(k)+v_{i}(k), \quad(i \in\{C V, C T\})
\end{aligned}
$$

where $x=\left[\begin{array}{llll}x_{1} & \dot{x}_{1} & x_{2} & \dot{x}_{2}\end{array}\right]^{T}$ where $x_{1}$ and $x_{2}$ are the position coordinates, $u=\left[\begin{array}{ll}u_{1} & u_{2}\end{array}\right]^{T}$ where $u_{1}$ and $u_{2}$ are the acceleration components. The control input has a different constant value for each mode:

$$
u_{C V}=\left[\begin{array}{l}
0 \\
0
\end{array}\right] \text { for CV mode, } u_{C T}=\left[\begin{array}{l}
1.5 \\
1.5
\end{array}\right] \text { for CT mode }
$$

where $T$ is the sampling interval and $w_{C V}, w_{C T}, v_{C V}$, and $v_{C T}$ are zero-mean, uncorrelated, white Gaussian process and measurement noise for the CV mode and the CT mode, respectively. An aircraft trajectory is shown in Figure 3 and the actual mode switches oc-

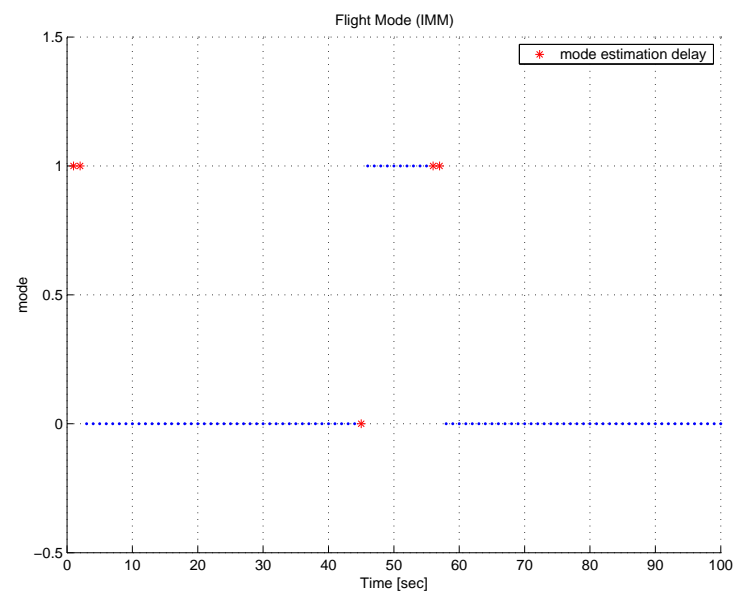

(a)

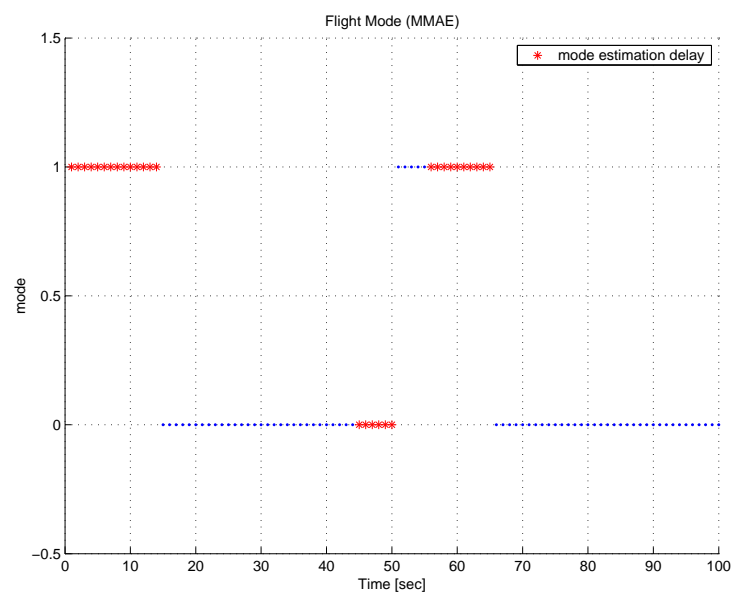

(b)

Figure 4: Aircraft mode estimates by (a) IMM and (b) MMAE: 100 trial Monte Carlo simulation results (mode $\mathrm{CV}=0$, mode $\mathrm{CT}=1$ ). 
cur at time $=45$ seconds $(\mathrm{CV}$ to $\mathrm{CT})$ and at time $=56$ seconds $(\mathrm{CT}$ to $\mathrm{CV})$. In this scenario, the aircraft behavior in the two modes is similar (i.e., the aircraft's yaw rate is small) in order to better demonstrate the performance of MMAE and IMM. Using Proposition 4.2 for IMM, we find that the mode estimation delay in switching from CV to CT is $\delta_{c t}=1$, and from $\mathrm{CT}$ to $\mathrm{CV}$ is $\delta_{c v}=2$. We therefore expect the mode switching from mode $\mathrm{CV}$ to $\mathrm{CT}$ to be more detectable than the mode switching from mode CT to CV. Similarly, we obtain $\delta_{c t}=7$ and $\delta_{c v}=12$ for MMAE. Figure 4 shows that the simulations validate these predictions well. IMM performs better than MMAE; and the mode estimation delays for both IMM (1 for CV to CT; 2 for CT to CV) and MMAE (6 for CV to CT; 10 for CT to $\mathrm{CV})$ are close to those predicted, and within the bounds.

False mode estimation could cause poor aircraft tracking and thus inaccurate trajectory prediction. This is undesirable for air traffic surveillance and control since conflict detection and resolution are based on the aircraft's current state estimate and the future trajectory prediction. This observation motivates the development of a hybrid estimation algorithm which could provide more accurate mode estimates than existing algorithms.

\section{Residual-Mean Interacting Multiple Model Algorithm (RMIMM)}

In this section, based on the performance analysis results in the previous section, we propose a modified IMM algorithm called the Residual-Mean Interacting Multiple Model (RMIMM) algorithm, which has a likelihood function that uses the properties of the mean of the residual produced by each Kalman filter.

As can be seen from the IMM algorithm in Section 3 and the performance analysis of hybrid estimation algorithms in Sections 4, the mode probability in (11) strongly depends on the likelihood function $\Lambda_{j}$. Thus, if the likelihoods of the modes are close to each other, the mode estimate may be inaccurate. Inaccurate mode estimates could produce poor state estimates. Therefore, we propose a method which reduces false mode estimation by increasing the difference between the likelihood of the correct mode and the likelihoods of the other modes, using the fact that if the Kalman filter corresponding to mode $j$ is the correct one, then the residual in (10) should be a white Gaussian process with a zero mean. Otherwise, its mean should not be zero. In this section, without loss of generality, we consider an autonomous discrete-time stochastic linear hybrid system (1)-(2), i.e., a system without control input $(u(k) \equiv 0)$. Then the mean of the residual is

$$
\bar{r}_{j}(k)=C_{T} A_{T} \bar{e}_{j}(k-1)+\left(C_{T} \Delta A_{j}+\Delta C_{j} A_{T}-\Delta C_{j} \Delta A_{j}\right) \hat{x}_{j}(k-1)
$$

and the mean of estimation error is 


$$
\bar{e}_{j}(k)=\left(I-K_{j}(k) C_{T}\right) A_{T} \bar{e}_{j}(k-1)+\left(\left(I-K_{j}(k) C_{T}\right) \Delta A_{j}-K_{j}(k) \Delta C_{j} A_{j}\right) \hat{x}_{k}(k-1)
$$

where $K_{j}(k)$ is the Kalman filter gain for Kalman filter $j$. This is a recursive equation with respect to the state estimation error. Thus, the mean of the residual is computed from (26) and (27).

To the best of our knowledge, all multiple-model-based estimation and learning algorithms including various IMM algorithms use a likelihood function whose mean is zero to determine the current mode in which the system lies $[22,23,7]$. We propose RMIMM, which uses the mean of the residual to increase the difference between the likelihood of the correct mode and those of the other modes, thereby decreasing the number of false mode estimates. In the IMM framework, we know only the mode probabilities i.e., we do not exactly know which model is the true model at any given time. Thus, we propose a new definition of the mean of the residual: a weighted sum of the mean of the residual computed by each Kalman filter with the mode probability estimate in (11) as the weight. Similarly, a new definition of the mean of the state estimation error is proposed as a weighted sum of the mean of the state estimation error corresponding to Kalman filter $j$ with the same weight.

$$
\begin{aligned}
\bar{r}_{j}(k):= & \sum_{j=1}^{N}\left\{C_{T} A_{T} \bar{e}_{j}(k-1)+\left(C_{T} \Delta A_{j}+\Delta C_{j} A_{T}-\Delta C_{j} \Delta A_{j}\right) \hat{x}_{j}(k-1)\right\} \mu_{j}^{-}(k) \\
\bar{e}_{j}(k):= & \sum_{j=1}^{N}\left\{\left(I-K_{j}(k) C_{T}\right) A_{T} \bar{e}_{j}(k-1)+\left(\left(I-K_{j}(k) C_{T}\right) \Delta A_{j}-K_{j}(k) \Delta C_{j} A_{j}\right)\right. \\
& \left.\times \hat{x}_{k}(k-1)\right\} \mu_{j}^{-}(k)
\end{aligned}
$$

If the mode probability of mode $j$ is large, the mean of the residual becomes small (i.e., close to zero) because the other mode probabilities $\mu_{i}(k)$ for $\forall i \neq j$ are small (the residual has a zero mean if the Kalman filter is the correct one). Since the proposed mean of the residual is small if the mode probability of the corresponding Kalman filter is small, and large if the mode probability of the corresponding Kalman filter is large, we can use the mean of the residual in (28) to make the likelihood of the correct mode more distinct from those of the other modes. Therefore, using the mean of the residual provided by each Kalman filter, we propose a new likelihood function:

$$
\Lambda_{j}^{\text {new }}(k)= \begin{cases}\frac{N_{j}(k) \Lambda_{j}(k)}{\sum_{i=1}^{N} N_{i}(k) \Lambda_{i}(k)} & \text { if } \bar{r}_{j}(k) \neq 0 \\ \Lambda_{j}(k) & \text { otherwise }\end{cases}
$$

where

$$
N_{i}(k)= \begin{cases}\left\|\bar{r}_{i}(k)\right\|^{-1} & \text { if } \bar{r}_{i}(k) \neq 0 \\ 1 & \text { otherwise }\end{cases}
$$

Proposition 5.1 The difference between the new likelihood function (29) for the correct mode and those for the incorrect mode, is greater than the corresponding difference using the previous likelihood function from (10). 
Proof If the model in Kalman filter $j$ is incorrect, the mean of residual is not zero and the likelihood of mode $j$ from the new likelihood function in (29) is less than that of the standard likelihood function in (10). If the model in Kalman filter $j$ is correct, the likelihood of mode $j$ from the new likelihood function is the same as that of the standard likelihood function in (10). Thus, the differences between the likelihood of the correct mode and those of incorrect modes are greater and the result follows.

We demonstrate the performance of the proposed RMIMM algorithm through an aircraft tracking example in which the aircraft trjectory is shown in Figure 1. We consider the acceleration model as the aircraft model for accurate aircraft tracking. Let the state of an aircraft defined as $x=\left[x_{1} \dot{x}_{1} \ddot{x}_{1} x_{2} \dot{x}_{2} \ddot{x}_{2}\right]^{T}$ where where $x_{1}$ and $x_{2}$ are the position coordinates. The aircraft model for CV mode is

$$
\begin{gathered}
x(k)=\left[\begin{array}{cccccc}
1 & T & 0 & 0 & 0 & 0 \\
0 & 1 & 0 & 0 & 0 & 0 \\
0 & 0 & 0 & 0 & 0 & 0 \\
0 & 0 & 0 & 1 & T & 0 \\
0 & 0 & 0 & 0 & 1 & 0 \\
0 & 0 & 0 & 0 & 0 & 0
\end{array}\right] x(k-1)+\left[\begin{array}{cc}
\frac{T^{2}}{2} & 0 \\
T & 0 \\
0 & 0 \\
0 & \frac{T^{2}}{2} \\
0 & T \\
0 & 0
\end{array}\right] w_{C V}(k) \\
y(k)=\left[\begin{array}{cccccc}
1 & 0 & 0 & 0 & 0 & 0 \\
0 & 0 & 0 & 1 & 0 & 0
\end{array}\right] x(k)+v_{C V}(k)
\end{gathered}
$$

For CT mode, the aircraft model (Wiener-sequence acceleration model) is

$$
\begin{gathered}
x(k)=\left[\begin{array}{cccccc}
1 & T & \frac{T^{2}}{2} & 0 & 0 & 0 \\
0 & 1 & T & 0 & 0 & 0 \\
0 & 0 & 1 & 0 & 0 & 0 \\
0 & 0 & 0 & 1 & T & \frac{T^{2}}{2} \\
0 & 0 & 0 & 0 & 1 & T \\
0 & 0 & 0 & 0 & 0 & 1
\end{array}\right] x(k-1)+\left[\begin{array}{cc}
\frac{T^{2}}{2} & 0 \\
T & 0 \\
1 & 0 \\
0 & \frac{T^{2}}{2} \\
0 & T \\
0 & 1
\end{array}\right] w_{C T}(k) \\
y(k)=\left[\begin{array}{cccccc}
1 & 0 & 0 & 0 & 0 & 0 \\
0 & 0 & 0 & 1 & 0 & 0
\end{array}\right] x(k)+v_{C T}(k)
\end{gathered}
$$

where $w_{C V}, w_{C T}, v_{C V}$, and $v_{C T}$ are zero-mean, uncorrelated, white Gaussian process noise and measurement noise for CV mode and CT mode, respectively. The following Markov discrete state (mode) transition matrix defined in (2) is used

$$
\Pi=\left[\begin{array}{ll}
0.95 & 0.05 \\
0.05 & 0.95
\end{array}\right]
$$


Here, the first column and the first row correspond to CV mode and the second column and
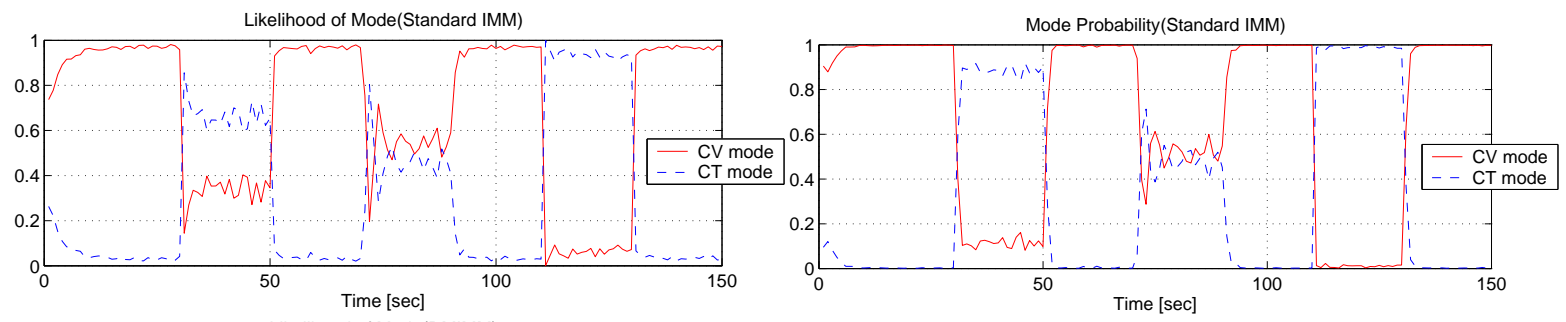

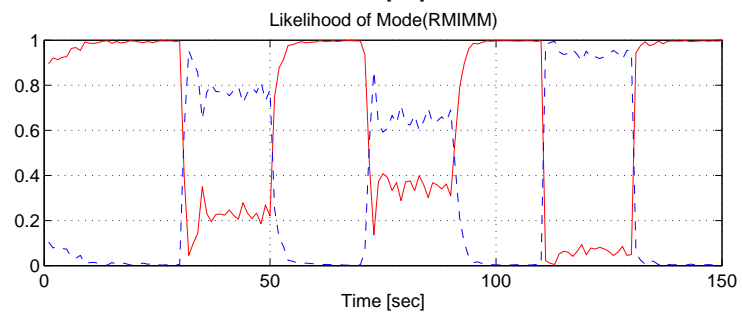

(a)

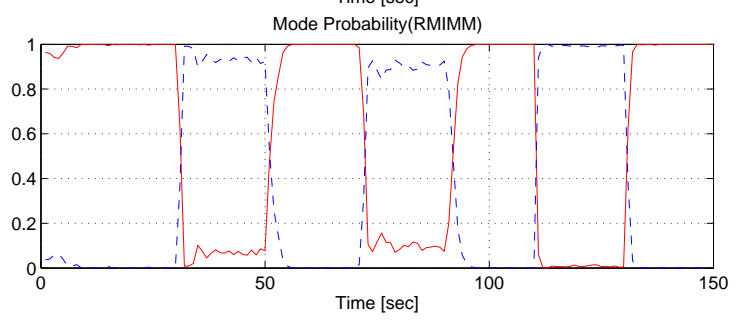

(b)

Figure 5: 100 times Monte Carlo simulation results using IMM and RMIMM for the aircraft tracking example. (a) Likelihood of each mode. (b) Mode probability of each mode.

the second row correspond to $\mathrm{CT}$ mode. For example, $\pi_{12}$ represents the mode transition probability from $\mathrm{CT}$ mode to $\mathrm{CV}$ mode. The mode transition matrix is a system parameter which represents the discrete dynamics of the system and the mode transition probability matrix in (30) has been chosen after many simulations.

We design a test flight trajectory with constant aircraft speed $v=480$ knots, composed of seven segments shown in Figure 1: straight flight from 0 to 30 seconds, a coordinated turn with $\omega=-3^{\circ} / \mathrm{sec}$ from 31 to 50 seconds, straight flight from 51 to 70 seconds, a coordinated turn with $\omega=1.5^{\circ} / \mathrm{sec}$ from 71 to 90 seconds, straight flight from 91 to 110 seconds, a coordinated turn with $\omega=-4.5^{\circ} / \mathrm{sec}$ from 111 to 130 seconds, and straight flight from 131 to 150 seconds. 100 trial Monte Carlo simulation results in Figure 5 show that RMIMM gives more distinct likelihoods of modes than those of the standard IMM algorithm. The RMS estimation errors of position and velocity using RMIMM are $15 \mathrm{~m}$ and $2.1 \mathrm{~m} / \mathrm{sec}$. The RMS estimation errors of position and velocity using IMM are $18 \mathrm{~m}$ and $2.3 \mathrm{~m} / \mathrm{sec}$. The RMS estimation errors of RMIMM are slightly better than those of IMM, yet both algorithms give smaller RMS errors than those of the raw measurements. Thus, the main advantage of RMIMM - that it gives better mode estimates than those of IMM is demonstrated. 


\section{Conclusions}

Several hybrid estimation algorithms have existed for many years. In this paper, we have performed a detailed steady-state and transient analysis of these algorithms and derived necessary conditions for correct mode detection, bounds on performance (in terms of the mode detection delay and the minimum sojourn time), and also proposed a way to predict a priori which mode transitions are the easiest to detect. We have validated our results using simulated experiments motivated from aircraft tracking problems. Our results give a mathematical yet intuitive explanation as to why the IMM algorithm achieves its high levels of performance in the estimation of stochastic linear hybrid systems, thus inspiring the development of new estimation algorithms. Based on the performance analysis results, a new variant of hybrid estimation algorithms, called the Residual-Mean Interacting Multiple Model (RMIMM) algorithm has been proposed and its performance has been demonstrated through a maneuvering aircraft tracking example.

\section{References}

[1] M.D. Lemmon, K.X. He, and I. Markovsky. Supervisory hybrid systems. IEEE Control Systems Magazine, 19(4):42-55, August 1999.

[2] C. Tomlin, G. Pappas, and S. Sastry. Conflict resolution for air traffic management: A study in multiagent hybrid systems. IEEE Transactions on Automatic Control, 43(4), 1998.

[3] B. Lennartson, M. Tittus, B. Egardt, and S. Petterson. Hybrid systems in process control. IEEE Control Systems Magazine, 16(5):45-56, October 1996.

[4] A. Balluchi, L. Benvenuti, M.D. di Benedetto, C. Pinello, and A.L. Sangiovanni-Vincentelli. Automotive engine control and hybrid systems: challenges and opportunities. Proceedings of the IEEE, 88(7):888-912, July 2000.

[5] P.S. Maybeck. Stochastic Models, Estimation, and Control, volume 2. New York: Academic Press, 1982.

[6] Y. Bar-Shalom, X.R. Li, and T. Kirubarajan. Estimation with Applications to Tracking and Navigation. John Wiley \& Sons, 2001.

[7] D.D. Sworder and J.E. Boyd. Estimation problems in Hybrid Systems. Cambridge University Press, 1999.

[8] R.M. Hawkes and J.B. Moore. Performance bounds for adaptive estimation. Proceedings of the IEEE, 64(8):1143-1150, August 1976.

[9] D.T. Magill. Optimal adaptive estimation of sampled stochastic processes. IEEE Transactions on Automatic Control, 10(4):434-439, October 1965.

[10] D.G. Lainiotis and F.L. Sims. Performance measures for adaptive Kalman estimators. IEEE Transactions on Automatic Control, 15(2):249-250, April 1970.

[11] F.L. Sims, D.G. Lainiotis, and D.T. Magill. Recursive algorithm for the calculation of the adaptive Kalman filter weighting coefficients. IEEE Transactions on Automatic Control, 14(2):215218, April 1969. 
[12] D.G. Lainiotis. Optimal adaptive estimation: Structure and parameter adaptation. IEEE Transactions on Automatic Control, 16(2):160-170, April 1971.

[13] Y. Baram and N.R. Sandell. Consistent estimation on finite parameter sets with application to linear system identification. IEEE Transactions on Automatic Control, 23(3):451-454, June 1978.

[14] Y. Baram. A sufficient condition for consistent discrimination between stationary Gaussian models. IEEE Transactions on Automatic Control, 23(5):958-960, October 1978.

[15] I. Hwang, H. Balakrishnan, and C. Tomlin. Observability criteria and estimator design for stochastic linear hybrid systems. In Proceedings of IEE European Control Conference, Cambridge, UK, September 2003.

[16] M.J. Caputi. A necessary condition for effective performace of the Multiple Model Adaptive Estimator. IEEE Transactions on Aerospace and Electronic Systems, 31(3):1132-1138, July 1995.

[17] X.R. Li and Y. Bar-Shalom. Design of an Interacting Multiple Model algorithm for Air Traffic Control tracking. IEEE Transactions on Control Systems Technology, 1(3):186-194, September 1993.

[18] H.A.P. Blom and Y. Bar-Shalom. The Interacting Multiple Model algorithm for systems with Markovian switching coefficients. IEEE Transactions on Automatic Control, 33(8):780-783, 1988.

[19] R.A. Horn. Matrix Analysis. Cambridge University Press, 1985.

[20] T. Kailath. Linear Systems. Prentice Hall, 1980.

[21] B. Etkin and L.D. Reid. Dynamics of Flight: Stability and Control. John Wiley \& Sons, 3 edition, 1996.

[22] E. Mazor, A. Averbuch, Y. Bar-Shalom, and J. Dayan. Interacting multiple model methods in target tracking: A survey. IEEE Transactions on Aerospace and electronic systems, 34(1):103123, January 1998.

[23] Y. Bar-Shalom and X.R. Li. Estimation and Tracking: Principles, Techniques, and Software. Artech House, Boston, 1993. 